\title{
Association of histone deacetylase expression with histology and prognosis of ovarian cancer
}

\author{
MITSUTAKE YANO $^{1,2}$, MASANORI YASUDA $^{1}$, MIKA SAKAKI $^{1}$, KOJI NAGATA $^{1}$, \\ TAKASHI FUJINO ${ }^{1}$, EIICHI ARAI ${ }^{1}$, TAKAHIRO HASEBE ${ }^{1}$, MASAKI MIYAZAWA ${ }^{3}$, \\ MARIKO MIYAZAWA $^{3}$, NAOKI OGANE ${ }^{4}$, KOSEI HASEGAWA ${ }^{5}$ and HISASHI NARAHARA ${ }^{2}$ \\ ${ }^{1}$ Department of Pathology, Saitama Medical University International Medical Center, \\ Saitama 350-1298; ${ }^{2}$ Department of Obstetrics and Gynecology, Oita University Faculty of Medicine, \\ Oita 879-5593; ${ }^{3}$ Department of Obstetrics and Gynecology, Tokai University School of Medicine, \\ Kanagawa 259-1193; ${ }^{4}$ Division of Pathology, Ashigarakami Hospital, Kanagawa 258-0003; ${ }^{5}$ Department of \\ Gynecologic Oncology, Saitama Medical University International Medical Center, Saitama 350-1298, Japan
}

Received August 15, 2017; Accepted November 20, 2017

DOI: $10.3892 / 01.2018 .7726$

\begin{abstract}
Histone deacetylase (HDAC) inhibitor is known to have a cytotoxic effect on ovarian cancer cell lines. The present study analyzed the association between immunohistochemical HDAC expression and clinicopathological findings, in particular, the association with histological type and effect of chemotherapy. The histology of the 201 ovarian cancers addressed was as follows: Serous carcinoma (SEC), 100 cases; clear cell carcinoma (CCC), 56 cases; endometrioid carcinoma (EMC), 36 cases; and mucinous carcinoma (MUC), 9 cases. Immunohistochemical analyses of HDACs 1, 2, 3, 4, 5, 6 and 7 expression levels were performed using tissue microarrays, composed of 201 primary tumors and 38 tumors following chemotherapy. Overexpression of HDAC1 was detected in the nucleus of all cases with MUC, followed by CCC (80\%), SEC (73\%), and EMC (53\%). CCC specifically demonstrated HDAC7 expression in both the nucleus (27\%) and the
\end{abstract}

Correspondence to: Professor Masanori Yasuda, Department of Pathology, Saitama Medical University International Medical Center, 1397-1 Yamane, Hidaka, Saitama 350-1298, Japan

E-mail: m_yasuda@saitama-med.ac.jp

Abbreviations: HDAC, histone deacetylase; SEC, serous carcinoma; CCC, clear cell carcinoma; EMC, endometrioid carcinoma; MUC, mucinous carcinoma; PFS, progression free survival; OS, overall survival; FIGO, International Federation of Obstetrics and Gynecology; TMA, tissue microarray; RT, room temperature; $\mathrm{CR}$, complete response; $\mathrm{PR}$, partial response; $\mathrm{SD}$, stable disease; PD, progressive disease; HR, hazard ratio; CI, confidence interval; CSC, cancer stem cell; HIF-1 $\alpha$, hypoxia inducible factor- $1 \alpha$; VEGF, vascular endothelial growth factor; PD-L1, programmed death-1 ligand

Key words: ovary, cancer, histone deacetylase, prognosis, chemotherapy resistance cytoplasm (54\%), and HDAC6 expression in the nucleus (34\%). The comparison between prior to and following chemotherapy revealed a nuclear expression increase in HDAC1 (76\% vs. $92 \% ; \mathrm{P}=0.03)$ and HDAC7 (0.0 vs. $16 \% ; \mathrm{P}=0.01)$, and cytoplasmic expression increase in HDAC6 (40 vs. 74\%; $\mathrm{P}=<0.01$ ) and HDAC7 (16 vs. 66\%; $\mathrm{P}=<0.01$ ). HDAC1 nuclear expression adversely affected overall survival in SEC $(\mathrm{P}=0.02)$ and EMC $(\mathrm{P}=0.03)$, and HDAC7 cytoplasmic expression in CCC was associated with a poor prognosis $(\mathrm{P}=0.06)$. In multivariate analysis, HDAC6 nuclear expression was determined as a poor prognostic factor (hazard ratio $=3.51 ; 95 \%$ confidence interval, 1.49 to $8.27, \mathrm{P}=<0.01$ ). In the subgroup analysis, HDAC6 nuclear expression was associated with a poor prognosis in CCC ( $\mathrm{P}=0.07)$, International Federation of Obstetrics and Gynecology stage III/IV $(\mathrm{P}=0.07)$, and suboptimal surgery $(\mathrm{P}=<0.01)$. In conclusion, HDACs may be associated with the prognosis of ovarian cancers, depending on the histological subtypes, and upregulated following chemotherapy. HDAC1, 6 and 7 may therefor act as promising therapeutic targets in the future.

\section{Introduction}

Ovarian cancer is the leading cause of death among gynecological malignancies, and is also the fourth most common malignancy in women in developed countries, following breast, lung, and colorectal cancer $(1,2)$. Apart from surgery and radiotherapy, a substantial number of ovarian cancer patients commonly undergo chemotherapy because of its high efficacy. However, some of these patients frequently develop varying degrees of chemotherapeutic resistance, which is closely associated with the histological subtypes. Each of the ovarian cancers, represented by serous carcinoma (SEC), endometrioid carcinoma (EMC), clear cell carcinoma (CCC) and mucinous carcinoma (MUC), is known to have a specific prognosis and chemotherapy sensitivity.

Histone deacetylases (HDACs) are chromatin-modifying enzymes that are involved in regulation of many aspects of 
cell biology, including tissue differentiation, autophagy, apoptosis, migration, mitosis and angiogenesis via deacetylation of histone or non-histone protein (3). The HDAC family contains 18 enzymes and is divided into four classes based on their sequences similar to the yeast. In terms of enzymatic activity, HDAC1, 2, 3 and 8 for class I, HDAC4, 5, 6, 7, 9 and 10 for class II, and HDAC11 for class IV are zinc-dependent, and SIRT1-SIRT7 for class III are NAD+ dependent. Class I HDACs are considered as nuclear proteins and class II HDACs shuttle between the nucleus and cytoplasm (4). In several cancers including ovarian cancer, class I HDACs are upregulated and high HDAC1 expression is associated with a poor prognosis (5-10). In vitro, pan-HDAC inhibitor has been demonstrated to have a cytotoxic effect for ovarian cancer cell lines (11). However, the clinical trials resulted in limited therapeutic effect of pan-HDAC inhibitor because of the side toxicity (12). Therefore, more selective and effective HDACs inhibitors are needed in the therapy for ovarian cancers.

This study was conducted to analyze the association between immunohistochemical HDACs expression and clinicopathological findings, especially focusing on histological subtypes, prognosis and chemotherapy, with the aim at exploration of the new possible therapeutic strategies for ovarian cancer.

\section{Materials and methods}

Patient data and clinicopathological features (Table I). Patient electronic medical charts from the Saitama Medical University International Medical Center from 2008 to 2012 were reviewed under approval of the Institutional Review Board (IRB) following the ethical standards of the responsible committee on human experimentation and with the Helsinki Declaration of 1975, as revised in 1983. A total of 201 epithelial ovarian cancer patients (SEC, 100 cases; CCC, 56 cases; EMC, 36 cases; MUC, 9 cases) without preoperative chemotherapy, whose tumors were surgically resected and pathologically confirmed, were recruited in this study. We also obtained the specimens of 38 tumors ( 34 for SEC; 1 for CCC; 3 for EMC) after chemotherapy in addition to before chemotherapy in the same case. Clinicopathological characteristics with these cases, such as age, menopause, parity, recurrence, death, progression free survival (PFS), overall survival (OS), and the International Federation of Obstetrics and Gynecology (FIGO) stage, and treatment methods were reviewed.

Immunohistochemistry staining. Immunohistochemical expression of HDACs was analyzed using tissue microarray (TMA: KIN-2; Azumaya, Tokyo, Japan) under approval of the IRB. TMA was generated from 2 cylindrical cores $3.0 \mathrm{~mm}$ in diameter in each block, which were punched out of paraffin-embedded tissue blocks corresponding to the representative histological findings and were inserted into a recipient block. The tissue blocks consisted of 201 cases with primary tumors which did not undergo neo-adjuvant chemotherapy. In addition, 38 tissue blocks in which the tumors had undergone chemotherapy were used. The total of 239 tissue blocks were cut into $4-\mu \mathrm{m}$ serial sections, which were run through an automated system by Dako Autostainer Link 48 (Agilent Technologies, Inc., Santa Clara, CA, USA) as per
Table I. Clinicopathological characteristics of patients $(n=201)$.

\begin{tabular}{|c|c|}
\hline Variable & No. $(\%)$ \\
\hline \multicolumn{2}{|l|}{ Age } \\
\hline Median (range) & $57.4(26-84)$ \\
\hline$<50$ & $57(28.4)$ \\
\hline$\geq 50$ & $144(71.6)$ \\
\hline \multicolumn{2}{|l|}{ Parity } \\
\hline$<2$ & 64 (31.8) \\
\hline$\geq 2$ & $137(68.2)$ \\
\hline \multicolumn{2}{|l|}{ Menopause } \\
\hline Yes & $57(28.3)$ \\
\hline No & $144(71.6)$ \\
\hline \multicolumn{2}{|l|}{ CA125 (U/ml) } \\
\hline Median (range) & $6,383.8(7-865,591)$ \\
\hline$\geq 200$ & $77(38.3)$ \\
\hline$<200$ & $124(61.7)$ \\
\hline \multicolumn{2}{|l|}{ Treatment } \\
\hline OP only & $24(11.9)$ \\
\hline $\mathrm{OP}+\mathrm{AC}$ & $88(43.8)$ \\
\hline $\mathrm{OP}+\mathrm{AC}+\mathrm{IDS}$ & $10(5.0)$ \\
\hline $\mathrm{NAC}+\mathrm{IDS}$ & $54(26.9)$ \\
\hline EL+Chemotherapy & $18(9.0)$ \\
\hline Others & $7(3.4)$ \\
\hline \multicolumn{2}{|l|}{ Histological subtype } \\
\hline Serous & $100(49.7)$ \\
\hline Clear cell & $56(27.9)$ \\
\hline Endometrioid & $36(17.9)$ \\
\hline Mucinous & $9(4.5)$ \\
\hline \multicolumn{2}{|l|}{ FIGO stage } \\
\hline I & $73(36.3)$ \\
\hline II & $24(11.9)$ \\
\hline III & $83(41.3)$ \\
\hline IV & $21(10.5)$ \\
\hline \multicolumn{2}{|l|}{ Recurrence } \\
\hline Yes & $106(52.7)$ \\
\hline No & $95(47.3)$ \\
\hline \multicolumn{2}{|l|}{ Death } \\
\hline Yes & $65(32.3)$ \\
\hline No & $136(67.7)$ \\
\hline
\end{tabular}

CA125, cancer antigen 125; OP, standard operation (at least hysterectomy, bilateral adnexectomy, and omentectomy); AC, adjuvant chemotherapy; IDS, interval debulking surgery; NAC, neo-adjuvant chemotherapy; EL, exploratory laparotomy; FIGO, International Federation of Gynecology and Obstetrics.

manufacturer's protocol. The primary antibodies used were as follows: Polyclonal rabbit anti-HDAC1 (dilution:15,000; ab19845; Abcam, Cambridge, UK); monoclonal rabbit anti-HDAC2 (dilution, 1:1,000; ab32117; Abcam); monoclonal rabbit anti-HDAC3 (dilution, 1:250; ab32369; Abcam); polyclonal rabbit anti-HDAC4 (dilution, 1:500; ab12172; Abcam); 

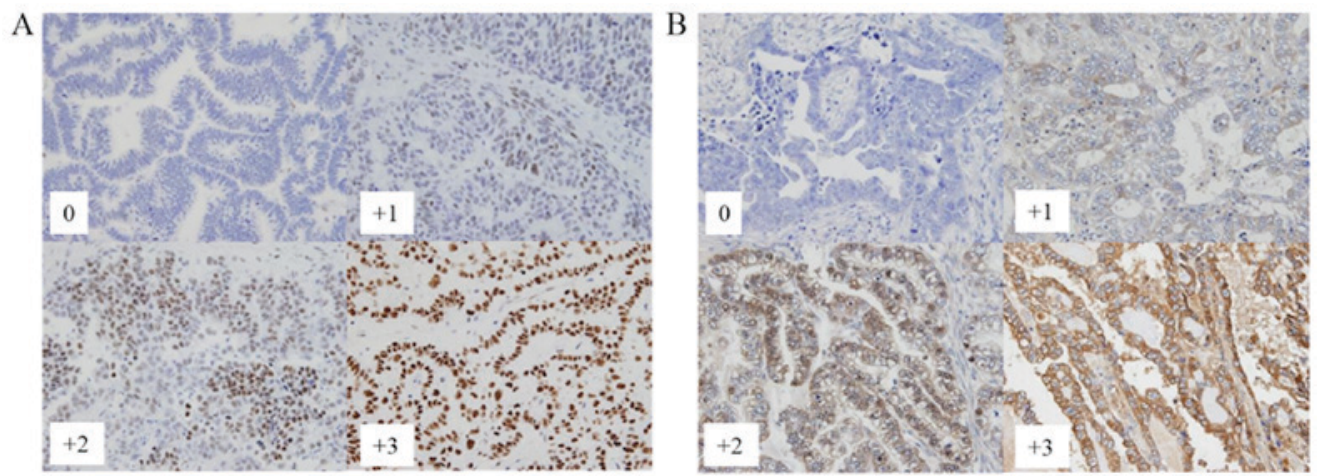

Figure 1. A four-tiered scoring system of immunohistochemical expressions. (A) Nuclear expression of HDAC1: 0 for negative cases; +1 for weak intensity; +2 for moderate intensity; and +3 for strong intensity. (B) Cytoplasmic expression of HDAC7: 0 for negative cases; +1 for weak intensity; +2 for moderate intensity; and +3 for strong intensity. HDAC, histone deacetylase.

A

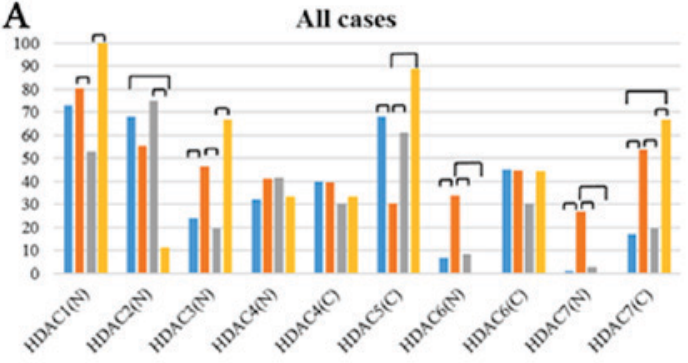

" Serous $(\mathrm{n}=100)=$ Clear $(\mathrm{n}=56)$ | Endometrioid $(\mathrm{n}=36)=$ Mucinous $(\mathrm{n}=9)$

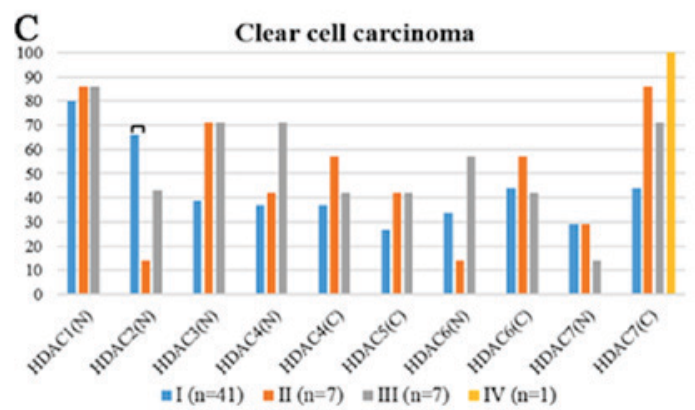

B

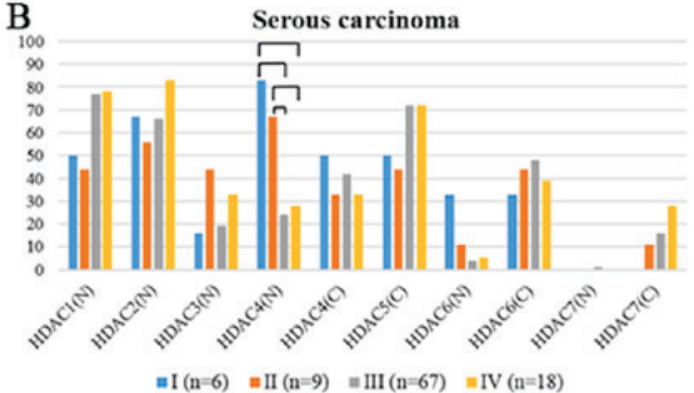

$\mathrm{D}$

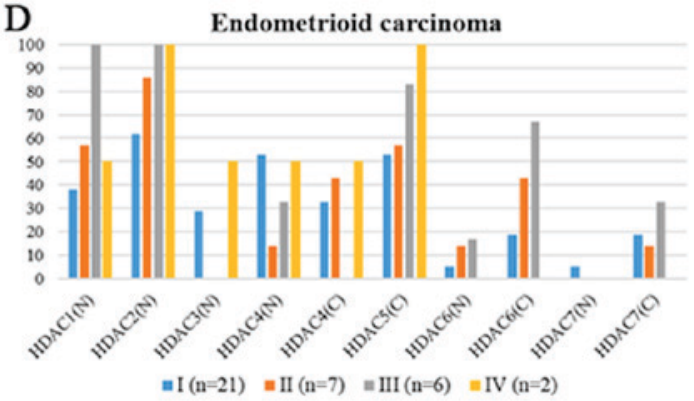

Figure 2. HDACs expression (\%) of each histological subtype in each FIGO stage: (A) HDACs expression (\%) of each histological subtypes in all FIGO stages. HDACs expression of each FIGO stage in (B) SEC, (C) CCC, and (D) EMC. P-values, Kruskal-Wallis tests. HDAC, histone deacetylase; FIGO, International Federation of Obstetrics and Gynecology; SEC, serous carcinoma; CCC, clear cell carcinoma; EMC, endometrioid carcinoma.

polyclonal rabbit anti-HDAC5 (dilution, 1:200; ab55403; Abcam); polyclonal rabbit anti-HDAC6 (dilution, 1:500; ab1440; Abcam); and polyclonal rabbit anti-HDAC7 (dilution, 1:100; NB100-61587; Novus Biological, Colorado, USA). For all antibodies but HDAC7, Target Retrieval Solution (pH 9.0) was applied for the antigen retrieval at $98^{\circ} \mathrm{C}$ for $20 \mathrm{~min}$. Sections were incubated with the primary antibodies at room temperature (RT) for $60 \mathrm{~min}$, followed by incubation with a secondary antibody (EnVision FLEX/HRP; Agilent Technologies, Inc.) at RT for $30 \mathrm{~min}$. The chromogen reaction was performed with diaminobenzidine plus the $\mathrm{H}_{2} \mathrm{O}_{2}$ substrate at $\mathrm{RT}$ for $10 \mathrm{~min}$. It was confirmed that there are no significant differences in all of HDACs expressions between TMA and the whole section, using 20 randomized cases (data not shown).

Interpretation of immunohistochemical results. Diagnoses were performed by one experienced pathologist who was blind to clinical data and patient characteristics, and one physician with a subspecialty in gynecological oncology. A four-tiered scoring scheme was used for both nuclear expression (Fig. 1A) and cytoplasmic expression (Fig. 1B), respectively: 0 for negative; +1 for weak; +2 for moderate; and +3 for marked. To optimize for PFS and OS differences, the raw data were binarized for statistical analysis as follows: The moderate $(+2)$ and marked $(+3)$ cases were grouped as high-level expressers, whereas the completely negative $(0)$ and weak $(+1)$ cases were considered as low-level expressers.

Statistical analysis. Univariable survival analysis was performed by the generation of Kaplan-Meier curves, and differences between the groups were assessed using the log rank statistic. Univariable and multivariable survival analysis was performed using the Cox proportional hazards model. Kruskal-Wallis tests were used to assess the change in the 


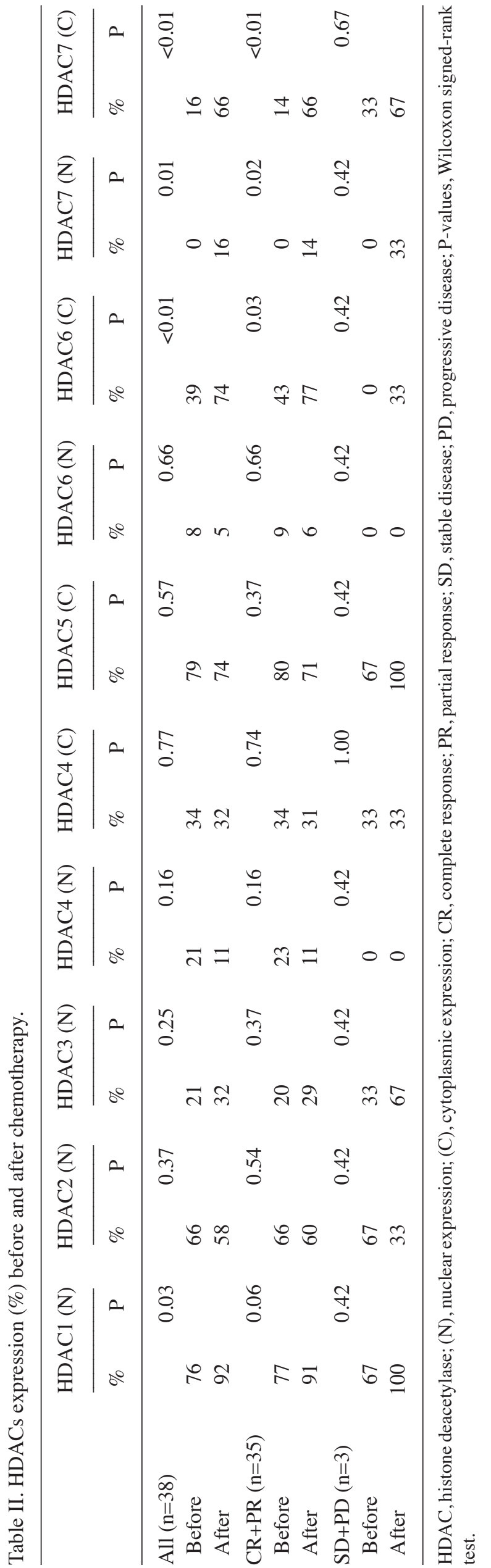

Table III. Univariable and multivariable analysis using the Cox proportional hazards model of overall survival for ovarian cancer ( $n=201 / 65$ events).

A, Univariate analysis

\begin{tabular}{lccr}
\hline Variables & HR & $95 \%$ Cl & P-value \\
\hline Age (>50 vs. s50) & 1.45 & $0.89-2.37$ & 0.13 \\
Histology & & & \\
$\quad$ Serous & 1.00 & & \\
Clear cell & 0.28 & $0.13-0.58$ & $<0.01$ \\
Endometrioid & 0.26 & $0.10-0.66$ & 0.04 \\
Mucinous & 1.00 & $0.36-2.78$ & 1.00 \\
Stage (III/IV vs. I/II) & 13.3 & $5.71-30.8$ & $<0.01$ \\
Surgery & 7.97 & $4.30-14.7$ & $<0.01$ \\
(optimal vs. suboptimal) & & & \\
HDAC1 (N) & 2.06 & $1.10-3.87$ & 0.02 \\
HDAC2 (N) & 1.25 & $0.75-2.08$ & 0.39 \\
HDAC3 (N) & 0.90 & $0.53-1.53$ & 0.69 \\
HDAC4 (N) & 0.85 & $0.51-1.43$ & 0.55 \\
HDAC4 (C) & 1.16 & $0.70-1.91$ & 0.57 \\
HDAC5 (C) & 2.54 & $1.46-4.42$ & $<0.01$ \\
HDAC6 (N) & 0.93 & $0.46-1.89$ & 0.85 \\
HDAC6 (C) & 1.18 & $0.72-1.91$ & 0.52 \\
HDAC7 (N) & 0.14 & $0.02-1.00$ & 0.05 \\
HDAC7 (C) & 1.19 & $0.70-1.99$ & 0.52 \\
& & &
\end{tabular}

B, Multivariate analysis

\begin{tabular}{lccr}
\hline Variables & HR & $95 \% \mathrm{Cl}$ & P-value \\
\hline $\begin{array}{l}\text { Stage (III/IV vs. I/II) } \\
\text { Surgery }\end{array}$ & 10.8 & $3.67-32.0$ & $<0.01$ \\
(optimal vs. suboptimal) & 11.1 & $3.32-37.4$ & $<0.01$ \\
Histology & & & \\
$\quad$ Serous & 1.00 & & \\
Clear cell & 8.11 & $2.33-28.3$ & $<0.01$ \\
Endometrioid & 2.43 & $0.94-6.30$ & 0.07 \\
$\quad$ Mucinous & 36.5 & $8.26-161$ & $<0.01$ \\
HDAC6(N) & 3.51 & $1.49-8.27$ & $<0.01$ \\
\end{tabular}

$\mathrm{HR}$, hazard ratio; $\mathrm{CI}$, indicates confidence interval; $(\mathrm{N})$, nuclear expression; (C), cytoplasmic expression.

distribution of HDAC expression across primary histological subtypes. Wilcoxon signed-rank test was used to assess the change between before and after chemotherapy. All analyses were performed using SPSS v24.0 (SPSS, Inc, Chicago, IL, USA). $\mathrm{P}<0.05$ was considered to indicate a statistically significant difference.

\section{Results}

Correlation of HDACs expression with histological subtype. HDACs overexpressions of each histological subtype are shown 

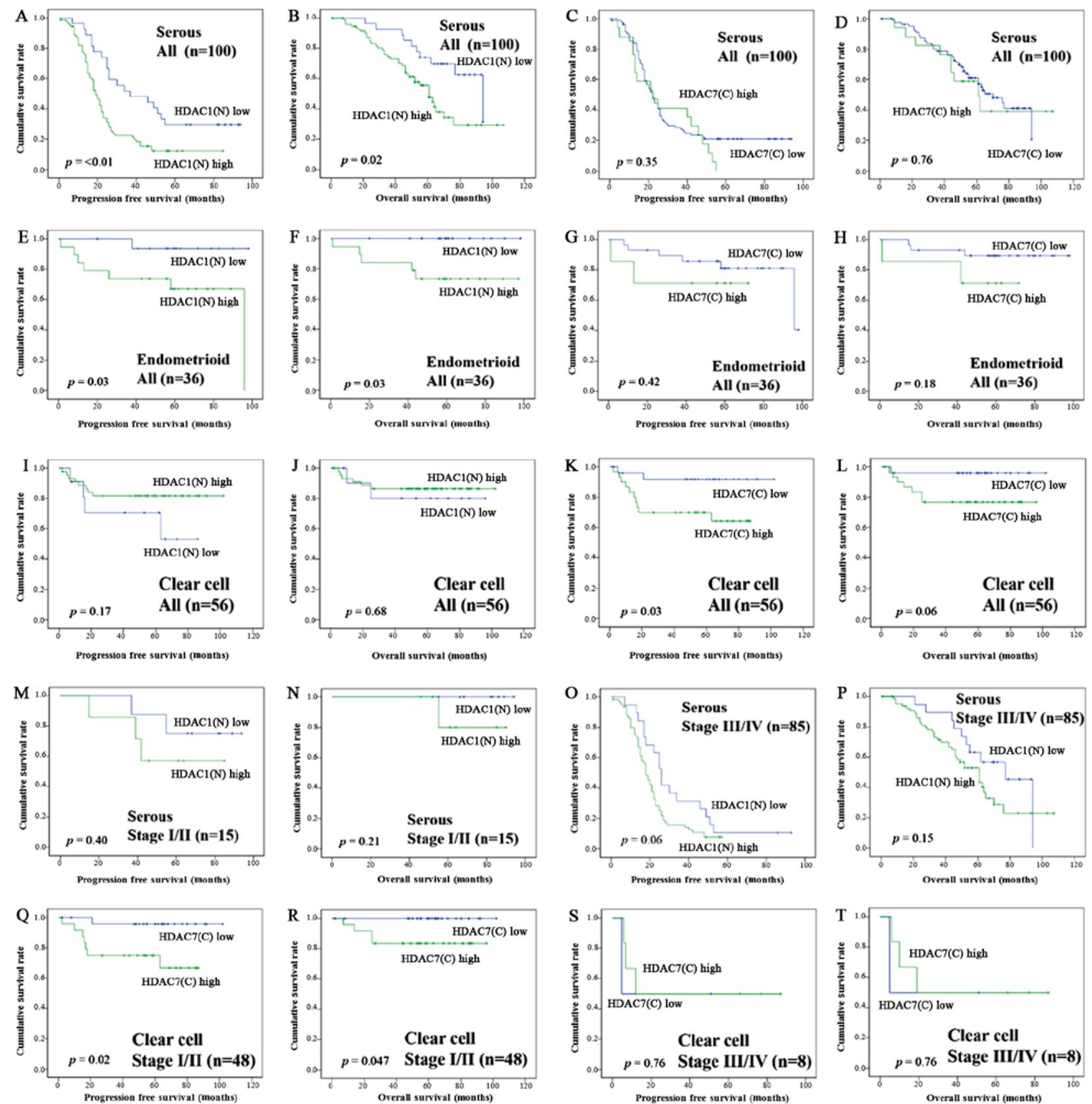

Figure 3. Kaplan-Meier survival analysis: Serous carcinoma patients according to the HDAC1 nuclear expression (A, PFS; B, OS) and HDAC7 cytoplasmic expression (C, PFS; D, OS). Endometrioid carcinoma patients according to the HDAC1 nuclear expression (E, PFS; F, OS) and HDAC7 cytoplasmic expression (G, PFS; H, OS). Clear cell carcinoma patients according to the HDAC1 nuclear expression (I, PFS; J, OS) and HDAC7 cytoplasmic expression (K, PFS; L, OS). Serous carcinoma patients according to the HDAC1 nuclear expression in FIGO stage I/II (M, PFS; N, OS) and stage III/IV (O, PFS; P, OS). Clear cell carcinoma patients according to the HDAC1 nuclear expression in FIGO stage I/II (Q, PFS; R, OS) and stage III/IV (S, PFS; T, OS). P-values, log-rank test. HDAC, histone deacetylase; PFS, progression free survival; OS, overall survival; FIGO, International Federation of Obstetrics and Gynecology.

in Fig. 2A. Expressions of HDAC1, 2, and 3 were observed only in the nucleus. Overexpression of HDAC1 was detected in all cases with MUC, followed by CCC (80\%), SEC (73\%), and EMC (53\%). HDAC2 expression was observed in EMC $(75 \%)$ and SEC (68\%). HDAC3 expression was done in MUC $(67 \%)$ and CCC (46\%). CCC showed the highest frequency of HDAC7 (27\%) and HDAC6 (34\%) expression in the nucleus among all the subtypes. On the other hand, CCC showed the lowest frequency of HDAC5 cytoplasmic expression (30\%). There were no significant differences in HDAC4 expression in both nucleus and cytoplasm and HDAC6 cytoplasmic expression among the histological subtypes. We analyzed the
HDACs expressions of each FIGO stage in SEC (Fig. 2B), CCC (Fig. 2C), and EMC (Fig. 2D). In SEC, FIGO stage I/II $(83 / 67 \%)$ showed higher frequency of HDAC4 nuclear expression than stage III/IV (24/28\%). In CCC, FIGO stage I (66\%) showed higher frequency of HDAC2 nuclear expression than stage II (14\%). There were no significant differences in other HDACs expression among each FIGO stage.

Correlation of HDACs expression with chemotherapy (Table II). The chemotherapy responses were evaluated as follows: 38 ovarian cancers clinically are 3 for complete response (CR), 31 for partial response (PR), 2 for stable disease 

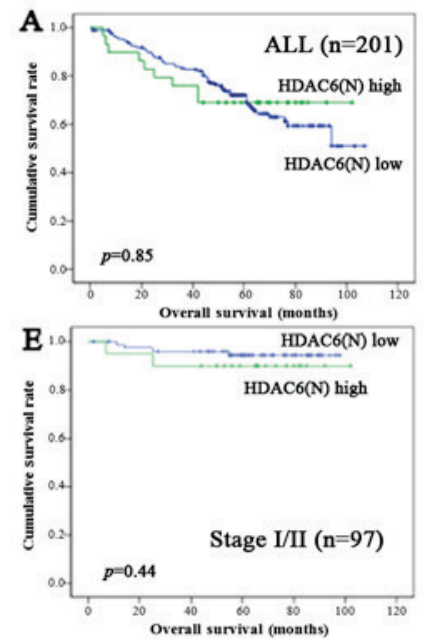
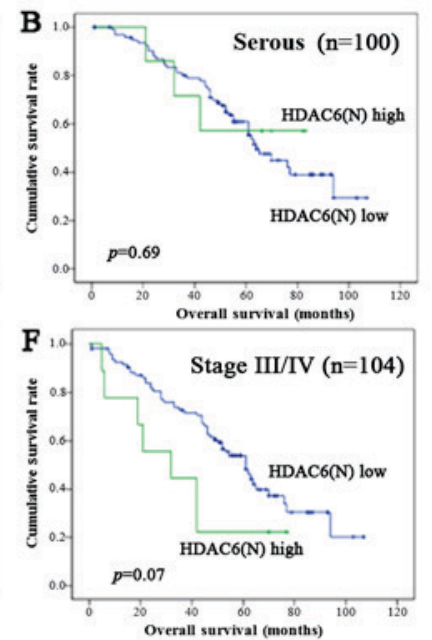
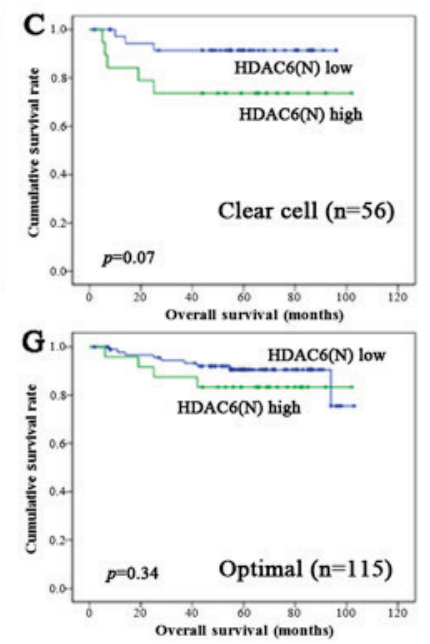
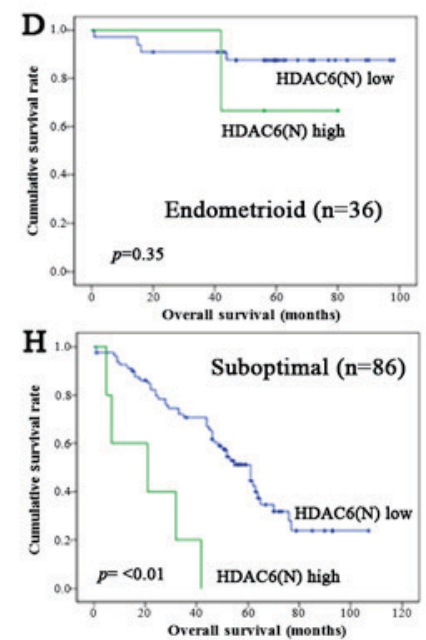

Figure 4. Kaplan-Meier survival analysis: OS according to the HDAC6 nuclear expression in all cases (A), SEC (B), CCC (C), EMC (D), FIGO stage I/II (E), stage III/IV (F), optimal surgery (G), and suboptimal surgery (H). P-values, log-rank test. OS, overall survival; HDAC, histone deacetylase; FIGO, International Federation of Obstetrics and Gynecology; SEC, serous carcinoma; CCC, clear cell carcinoma; EMC, endometrioid carcinoma.

(SD), and 2 for progressive disease (PD). In the comparison between before and after chemotherapy, HDAC1 nuclear expression increased from 76 to $92 \%(\mathrm{P}=0.03)$; HDAC7 expression in nucleus from 0 to $16 \%(\mathrm{P}=0.01)$ in cytoplasm from 16 to $66 \%(\mathrm{P}=<0.01)$; HDAC6 cytoplasmic expression increased from 39 to $74 \%(\mathrm{P}=<0.01)$. No significant changes were noted in other types of HDAC. We analyzed the CR+PR group ( $=35)$ and SD+PD group $(n=3)$, and found that in the $\mathrm{CR}+\mathrm{PR}$ group, HDAC1, 6, and 7 nuclear expressions and HDAC7 cytoplasmic expression increased in the comparison between before and after chemotherapy. In PD+PR group, no significant changes were noted in all types of HDAC.

Correlation of HDACs expression with prognosis. In SEC, overexpression of HDAC1 in the nucleus was significantly associated with the decrease in PFS ( $\mathrm{P}=<0.01$, Fig. $3 \mathrm{~A})$ and $\mathrm{OS}(\mathrm{P}=0.02$, Fig. 3B), but overexpression of HDAC7 in the cytoplasm had no significant adverse effect for PFS $(\mathrm{P}=0.35$, Fig. 3C) and $\mathrm{OS}$ ( $\mathrm{P}=0.76$, Fig. 3D). Also in EMC, overexpression of HDAC1 in the nucleus was significantly associated with the decrease in PFS ( $\mathrm{P}=0.03$, Fig. $3 \mathrm{E})$ and $\mathrm{OS}(\mathrm{P}=0.03$, Fig. 3F), but overexpression of HDAC7 in the cytoplasm had no significant adverse effect for PFS (P=0.42, Fig. $3 \mathrm{G}$ ) and OS $(\mathrm{P}=0.18$, Fig. $3 \mathrm{H})$. In $\mathrm{CCC}$, however, nuclear expression of HDAC1 showed no significant adverse effect for PFS $(\mathrm{P}=0.17$, Fig. 3I) and $\mathrm{OS}(\mathrm{P}=0.68$, Fig. $3 \mathrm{~J})$; on the other hand, cytoplasmic expression of HDAC7 was correlated with poor prognostic factor (PFS, $\mathrm{P}=0.03$; OS, $\mathrm{P}=0.06$, Fig. $3 \mathrm{~K}, \mathrm{~L}$ ). In the analysis focusing on the subgroup of FIGO stage I/II and stage III/IV in SEC and CCC (Fig. 3M-T), SEC patients with HDAC1 nuclear overexpression tended to have a poor prognosis in FIGO stage III/IV (PFS, $\mathrm{P}=0.06$; OS, $\mathrm{P}=0.15$ ), but had no significant effect on a prognosis in stage I/II (PFS, $\mathrm{P}=0.40$; OS, $\mathrm{P}=0.21$ ). CCC patients with HDAC7 cytoplasmic overexpression showed a poor prognosis in FIGO stage I/II (PFS, $\mathrm{P}=0.02$; OS, $\mathrm{P}=0.047$ ), but had no significant effect on a prognosis in stage III/IV (PFS, $\mathrm{P}=0.76$; OS, $\mathrm{P}=0.76$ ). HDAC5 cytoplasmic expression in EMC was associated with poor prognosis ( $\mathrm{PFS}, \mathrm{P}=0.01$; $\mathrm{OS}, \mathrm{P}=0.05$ ). HDAC4 nuclear expression in SEC was associated with longer PFS $(\mathrm{P}=0.03)$, but no significant change of OS $(\mathrm{P}=0.13)$. HDAC2, 3 , and 6 in each of the histological types had no significant effect on the prognosis.

Univariate and multivariate analyses (Table III). In univariate analysis, OS was associated with histological subtype, FIGO stage, surgical residual tumor, HDAC1 nuclear expression (hazard ratio $(\mathrm{HR})=2.06 ; 95 \%$ confidence interval $(\mathrm{CI})=1.10$ to 3.87; $\mathrm{P}=0.02)$ and HDAC5 cytoplasmic expression $(\mathrm{HR}=2.54$; $95 \% \mathrm{CI}, 1.46$ to $4.42 ; \mathrm{P}=<0.01)$. In multivariate survival analysis performed under inclusion of age, histological subtype, FIGO stage, surgical residual tumor, and HDACs expression, FIGO stage ( $\mathrm{HR}=10.8 ; 95 \% \mathrm{CI}, 3.67$ to $32.0 ; \mathrm{P}=<0.01)$, surgical residual tumor $(\mathrm{HR}=11.1 ; 95 \% \mathrm{CI}, 3.32$ to $37.4 ; \mathrm{P}=<0.01)$, histological subtype, and HDAC6 nuclear expression $(\mathrm{HR}=3.51$; 95\% CI, 1.49 to $8.27, \mathrm{P}=<0.01$ ) were found to become the independent prognostic factors. In the analysis with the subgroup of HDAC6 nuclear expression (Fig. 4), overexpression of HDAC6 in the nucleus had no significant adverse effect for $\mathrm{OS}$ in all cases $(\mathrm{P}=0.85$, Fig. 4A), $\mathrm{SEC}(\mathrm{P}=0.69$, Fig. 4B), EMC (P=0.35, Fig. 4D), FIGO stage I/II ( $\mathrm{P}=0.44$, Fig. 4E), and optimal surgery $(\mathrm{P}=0.34$, Fig. $4 \mathrm{G})$, but was associated with the decrease OS in CCC ( $\mathrm{P}=0.07$, Fig. $4 \mathrm{C})$, FIGO stage III/IV $(\mathrm{P}=0.07$, Fig. $4 \mathrm{~F})$, and suboptimal surgery $(\mathrm{P}=<0.01$, Fig. $4 \mathrm{H})$.

\section{Discussion}

It has been reported that class I HDACs are upregulated and high HDAC1 expression is associated with poor prognosis in several cancers including ovarian cancer (5-10). Class I HDACs are involved in regulation of many aspects of cancer biology including cell proliferation via p21, p27, and p57 $(13,14)$, apoptosis via p53, bcl2, caspase-3, -8 , and -9 (15), metastasis via e-cadherin (10), angiogenesis via hypoxia inducible factors- $1 \alpha$ (HIF-1 $\alpha)$ and vascular endothelial growth factor (VEGF) $(16,17)$, and anti-tumor immune responses via programmed death-1 ligand (PD-L1) (18). Weichert et al reported that overexpression of HDAC1 was an independent 
prognostic factor in ovarian EMC (9); however, the multivariable analysis does not contain the key prognostic factor in ovarian cancer, such as surgical debulking status (optimal or suboptimal). Hayashi et al (10) reported that overexpression of HDAC1 might be correlated with a poor prognosis in ovarian cancer, but did not analyze each of the histological subtypes in detail. Additionally, those two previous studies had not conducted an evaluation about class II HDACs. The present study was designed to supplement the deficiency in the previous studies and showed HDAC1 overexpression is a poor prognostic factor not only in EMC but also in SEC In CCC, HDAC6 and HDAC7 expressions were upregulated in comparison with other histological subtypes, and that HDAC7 cytoplasmic expression is expected to become a poor prognostic factor. Although HDAC6 nuclear expression had no significant effect on a prognosis in univariate analysis, it was found to have the significant as a poor prognostic factor in multivariate analysis employing FIGO stage, histological subtype, and surgical debulking status. By subgroup analysis, we found that HDAC6 nuclear overexpression is associated with a poor prognosis especially in surgical suboptimal cases. In SEC, the most prominent molecular changes include the alternation in TP53, which was exclusively mutations. HDAC1 provides the major enzymes for $\mathrm{p} 53$ deacetylation and form a Snail1/HDAC1/p53 tri-molecular complex, and inactivates p53 $(14,19,20)$. On the other hand, in CCC, ARIDIA mutation is the most common event (57\%) (21) and frequently coexists with PIK3CA mutation (22). HDAC6 activity is essential in ARIDIA-mutated ovarian cancers and HDAC6 inhibition selectively promoted apoptosis of ARIDIA-mutated cells (23). CCC is associated with Lynch syndrome, which is characterized by germline mutations in MSH2 $(24,25)$. HDAC6 deacetylates and ubiquitinates MSH2, causing a cellular tolerance to DNA damage and decreased cellular DNA mismatch repair activities (26). CCC is at a higher level of HIF-1 $\alpha$ than other histological subtypes (27), and HDAC7 increases transcriptional activity of HIF-1 $\alpha$ (28). In is suggested that the different HDAC isoforms may become a prognostic factor in SEC, EMC, and CCC. HDAC6 and 7 have a potential of being a chemotherapeutic target specifically for CCC.

HDAC1 and HDAC7 increased after chemotherapy. Residual tumor cells after neo-adjuvant chemotherapy might have low sensitivity or acquired resistance for chemotherapy. HDAC1 and HDAC7 augment cancer stem cell (CSC) phenotype via MiR-34a and the CSC markers such as CD44 and CD166, and the CSC phenotype is associated with chemotherapy resistance, metastasis, and relapse $(29,30)$. HDAC1 directly deacetylates HIF-1 $\alpha$ and blocks degradation of the protein (16). HDAC7 increases transcriptional activity of HIF- $1 \alpha$ through the formation of a complex with HIF-1 $\alpha$, HDAC7, and p300 in the nucleus (28). Overexpression of HIF-1 $\alpha$ reduced cisplatin-induced apoptosis in cisplatin-sensitive cells (31). HDAC inhibitor has been reported to have synergistic cytotoxicity with cisplatin in ovarian carcinoma cells and can restore cisplatin sensitivity in the acquired cisplatin-resistant cells $(15,32)$. HDAC1 and 7 have a potential of being a chemotherapeutic target for ovarian cancer with chemoresistance. It would be useful to clarify the correlation between HDACs and chemoresistance-related substances, such as HIF-1 $\alpha$, CD44, CD166, e-cadherin, MSH2, and PD-L1 etc. A potential weakness of the present study is the small population of several important subgroups, such as EMC $(n=36)$, MUC $(n=9)$, chemotherapy SD+PD group $(n=3)$. Further studies are needed to clarify the precise associations with those factors

In conclusion, this immunohistochemical study of HDACs expression revealed the correlation between the HDAC isoforms and the prognosis and histological subtypes. Further studies, especially focusing on HDAC1, 6, and 7, are needed in order to explore the strategy for histological subtypes of ovarian cancer with chemoresistance or low chemo-sensitivity.

\section{Acknowledgements}

This study was supported by the Hidaka Research Projects in Saitama Medical University (grant no. 28-D-1-14) and Grants-in-Aid from the Ministry of Education, Science, Sports and Culture of Japan (Research Project no. 16K11157). We thank Koichi Kamada and Tomomi Kato, Department of Pathology, Saitama Medical University International Medical Center, for their technical support.

\section{References}

1. Permuth-Wey J and Sellers TA: Epidemiology of ovarian cancer. Methods Mol Biol 472: 413-437, 2009.

2. Gayther SA and Pharoah PD: The inherited genetics of ovarian and endometrial cancer. Curr Opin Genet Dev 20: 231-238, 2010.

3. Seto E and Yoshida M: Erasers of histone acetylation: The histone deacetylase enzymes. Cold Spring Harb Perspect Biol 6: a018713, 2014.

4. Verdin E, Dequiedt F and Kasler HG: Class II histone deacetylases: Versatile regulators. Trends Genet 19: 286-293, 2003.

5. Higashijima J, Kurita N, Miyatani T, Yoshikawa K, Morimoto S, Nishioka M, Iwata T and Shimada M: Expression of histone deacetylase 1 and metastasis-associated protein 1 as prognostic factors in colon cancer. Oncol Rep 26: 343-348, 2011.

6. Miyake K, Yoshizumi T, Imura S, Sugimoto K, Batmunkh E, Kanemura $\mathrm{H}$, Morine $\mathrm{Y}$ and Shimada M: Expression of hypoxia-inducible factor-1alpha, histone deacetylase 1 , and metastasis-associated protein 1 in pancreatic carcinoma: Correlation with poor prognosis with possible regulation. Pancreas 36: e1-e9, 2008.

7. Minamiya Y, Ono T, Saito H, Takahashi N, Ito M, Mitsui M, Motoyama S and Ogawa J: Expression of histone deacetylase 1 correlates with a poor prognosis in patients with adenocarcinoma of the lung. Lung Cancer 74: 300-304, 2011.

8. Khabele D, Son DS, Parl AK, Goldberg GL, Augenlicht LH, Mariadason JM and Rice VM: Drug-induced inactivation or gene silencing of class I histone deacetylases suppresses ovarian cancer cell growth: Implications for therapy. Cancer Biol Ther 6: 795-801, 2007.

9. Weichert W, Denkert C, Noske A, Darb-Esfahani S, Dietel M, Kalloger SE, Huntsman DG and Köbel M: Expression of class I histone deacetylases indicates poor prognosis in endometrioid subtypes of ovarian and endometrial carcinomas. Neoplasia 10: 1021-1027, 2008

10. Hayashi A, Horiuchi A, Kikuchi N, Hayashi T, Fuseya C, Suzuki A, Konishi I and Shiozawa T: Type-specific roles of histone deacetylase (HDAC) overexpression in ovarian carcinoma: HDAC1 enhances cell proliferation and HDAC3 stimulates cell migration with downregulation of E-cadherin. Int J Cancer 127: 1332-1346, 2010.

11. Sonnemann J, Gänge J, Pilz S, Stötzer C, Ohlinger R, Belau A, Lorenz $\mathrm{G}$ and Beck JF: Comparative evaluation of the treatment efficacy of suberoylanilide hydroxamic acid (SAHA) and paclitaxel in ovarian cancer cell lines and primary ovarian cancer cells from patients. BMC Cancer 6: 183, 2006. 
12. Modesitt SC, Sill M, Hoffman JS and Bender DP; Gynecologic Oncology Group: A phase II study of vorinostat in the treatment of persistent or recurrent epithelial ovarian or primary peritoneal carcinoma: A Gynecologic Oncology Group study. Gynecol Oncol 109: 182-186, 2008.

13. Yamaguchi T, Cubizolles F, Zhang Y, Reichert N, Kohler H, Seiser C and Matthias P: Histone deacetylases 1 and 2 act in concert to promote the G1-to-S progression. Genes Dev 24: 455-469, 2010.

14. Zupkovitz G, Grausenburger R, Brunmeir R, Senese S, Tischler J, Jurkin J, Rembold M, Meunier D, Egger G, Lagger S, et al: The cyclin-dependent kinase inhibitor p21 is a crucial target for histone deacetylase 1 as a regulator of cellular proliferation. Mol Cell Biol 30: 1171-1181, 2010.

15. Hwang JJ, Kim YS, Kim MJ, Jang S, Lee JH, Choi J, Ro S, Hyun YL, Lee JS and Kim CS: A novel histone deacetylase inhibitor, CG0006, induces cell death through both extrinsic and intrinsic apoptotic pathways. Anticancer Drugs 20: 815-821, 2009.

16. Yoo YG, Kong G and Lee MO: Metastasis-associated protein 1 enhances stability of hypoxia-inducible factor-1alpha protein by recruiting histone deacetylase 1. EMBO $\mathrm{J} 25$ : 1231-1241, 2006

17. Ray A, Alalem M and Ray BK: Loss of epigenetic Kruppel-like factor 4 histone deacetylase (KLF-4-HDAC)-mediated transcriptional suppression is crucial in increasing vascular endothelial growth factor (VEGF) expression in breast cancer. J Biol Chem 288: 27232-27242, 2013.

18. Woods DM, Sodre AL, Villagra A, Sarnaik A, Sotomayor EM and Weber J: HDAC Inhibition upregulates PD-1 ligands in melanoma and augments immunotherapy with PD-1 blockade. Cancer Immunol Res 3: 1375-1385, 2015.

19. Yang H, Yan B, Liao D, Huang S and Qiu Y: Acetylation of HDAC1 and degradation of SIRT1 form a positive feedback loop to regulate p53 acetylation during heat-shock stress. Cell Death Dis 6: e1747, 2015.

20. Ni T, Li XY, Lu N, An T, Liu ZP, Fu R, Lv WC, Zhang YW, $\mathrm{Xu} \mathrm{XJ}$, Grant Rowe $\mathrm{R}$, et al: Snail1-dependent p53 repression regulates expansion and activity of tumour-initiating cells in breast cancer. Nat Cell Biol 18: 1221-1232, 2016.

21. Jones S, Wang TL, Shih IeM, Mao TL, Nakayama K, Roden R, Glas R, Slamon D, Diaz LA Jr, Vogelstein B, et al: Frequent mutations of chromatin remodeling gene ARID1A in ovarian clear cell carcinoma. Science 330: 228-231, 2010.

22. Yamamoto S, Tsuda H, Takano M, Tamai S and Matsubara O: Loss of ARID1A protein expression occurs as an early event in ovarian clear-cell carcinoma development and frequently coexists with PIK3CA mutations. Mod Pathol 25: 615-624, 2012.
23. Bitler BG, Wu S, Park PH, Hai Y, Aird KM, Wang Y, Zhai Y, Kossenkov AV, Vara-Ailor A, Rauscher FJ III, et al: ARID1A-mutated ovarian cancers depend on HDAC6 activity. Nat Cell Biol 19: 962-973, 2017.

24. Ketabi Z, Bartuma K, Bernstein I, Malander S, Grönberg H, Björck E, Holck S and Nilbert M: Ovarian cancer linked to Lynch syndrome typically presents as early-onset, non-serous epithelial tumors. Gynecol Oncol 121: 462-465, 2011.

25. Jensen KC, Mariappan MR, Putcha GV, Husain A, Chun N, Ford JM, Schrijver I and Longacre TA: Microsatellite instability and mismatch repair protein defects in ovarian epithelial neoplasms in patients 50 years of age and younger. Am J Surg Pathol 32: 1029-1037, 2008.

26. Zhang M, Xiang S, Joo HY, Wang L, Williams KA, Liu W, Hu C, Tong D, Haakenson J, Wang C, et al: HDAC6 deacetylates and ubiquitinates MSH2 to maintain proper levels of MutS $\alpha$. Mol Cell 55: 31-46, 2014.

27. Miyazawa M, Yasuda M, Fujita M, Hirasawa T, Kajiwara H, Hirabayashi K, Ogane N, Shimizu M, Asanuma H, Murakami M, et al: Association of hypoxia-inducible factor-1 expression with histology in epithelial ovarian tumors: A quantitative analysis of HIF-1. Arch Gynecol Obstet 279: 789-796, 2009.

28. Kato H, Tamamizu-Kato $\mathrm{S}$ and Shibasaki F: Histone deacetylase 7 associates with hypoxia-inducible factor lalpha and increases transcriptional activity. J Biol Chem 279: 41966-41974, 2004.

29. Witt AE, Lee CW, Lee TI, Azzam DJ, Wang B, Caslini C, Petrocca F, Grosso J, Jones M, Cohick EB, et al: Identification of a cancer stem cell-specific function for the histone deacetylases, HDAC1 and HDAC7, in breast and ovarian cancer. Oncogene 36: 1707-1720, 2017.

30. Wu MY, Fu J, Xiao X, Wu J and Wu RC: MiR-34a regulates therapy resistance by targeting HDAC1 and HDAC7 in breast cancer. Cancer Lett 354: 311-319, 2014.

31. Ai Z, Lu Y, Qiu S and Fan Z: Overcoming cisplatin resistance of ovarian cancer cells by targeting HIF-1-regulated cancer metabolism. Cancer Lett 373: 36-44, 2016.

32. Lin CT, Lai HC, Lee HY, Lin WH, Chang CC, Chu TY, Lin YW, Lee KD and Yu MH: Valproic acid resensitizes cisplatin-resistant ovarian cancer cells. Cancer Sci 99: 1218-1226, 2008.

This work is licensed under a Creative Commons Attribution-NonCommercial-NoDerivatives 4.0 International (CC BY-NC-ND 4.0) License. 